\title{
Ranking in-orbit fragmentations and space objects
}

\author{
Alessandro Rossi, ${ }^{1}$ Giovanni B. Valsecchi ${ }^{2,1}$ and Elisa Maria Alessi ${ }^{1}$ \\ ${ }^{1}$ IFAC-CNR \\ Via Madonna del Piano 10, 50019, Sesto Fiorentino, Italy \\ email: a.rossi@ifac.cnr.it, em.alessi@ifac.cnr.it \\ ${ }^{2}$ IAPS-INAF \\ via Fosso del Cavaliere 100, 00133, Roma, Italy \\ email: giovanni@iaps.inaf.it
}

\begin{abstract}
The future space debris environment will be dominated by the production of fragments coming from massive fragmentations. In order to identify the most relevant parameters influencing the long term evolution of the environment and to assess the criticality of selected space objects in different regions of the circumterrestrial space, a large parametric study was performed. In this framework some indicators were produced to quantify and rank the relevance of selected fragmentations on the long term evolution of the space debris population. Based on the results of the fragmentation studies, a novel analytic index, the Criticality of Spacecraft Index, aimed at ranking the environmental criticality of abandoned objects in LEO, has been devised and tested on a sample population of orbiting objects.
\end{abstract}

Keywords. celestial mechanics, space debris, fragmentation, space objects ranking

\section{Introduction}

The simulations of the long term evolution of the space debris population show how the future environment will be dominated by the production of fragments coming from massive fragmentations. A first warning in this respect came from the well known collision between Iridium 33 and Cosmos 2251 in 2009 which injected thousands of fragments larger than $1 \mathrm{~cm}$ into long lasting orbits in the most crowded zone of LEO, around $800-900 \mathrm{~km}$ of altitude. In this respect, it is of paramount importance to understand the consequences on the environment of a possible future large fragmentation of a given spacecraft in different regions of space.

This kind of study was performed in the framework of the ESA-ESOC Contract " $A s$ sessment Study for Fragmentation Consequence Analysis for LEO and GEO Orbits", taking the present distribution of intact objects as a proxy to identify the space objects more prone to future catastrophic collisions. Using SDM 4.2, the latest version of the long term LEO to GEO debris environment evolution model developed by our research group at the Italian National Research Council (CNR) in the past decades (Rossi et al. 2009), a reference long term evolution scenario, where the space activities are performed in a way similar to the one adopted in the last decade, was simulated for a time span of 200 years. On top of this reference scenario a number of different spacecraft were supposed to fragment in selected epochs. Comparing the long term evolution in the cases with and without the additional fragments generated by the artificially introduced fragmentation, the effect of this particular fragmentation on the environment was evaluated.

Performing this kind of comparisons over many spacecraft with different masses and orbital elements allows to understand the effects of selected typical fragmentations on the long term evolution of the debris population, as a function of the main driving 


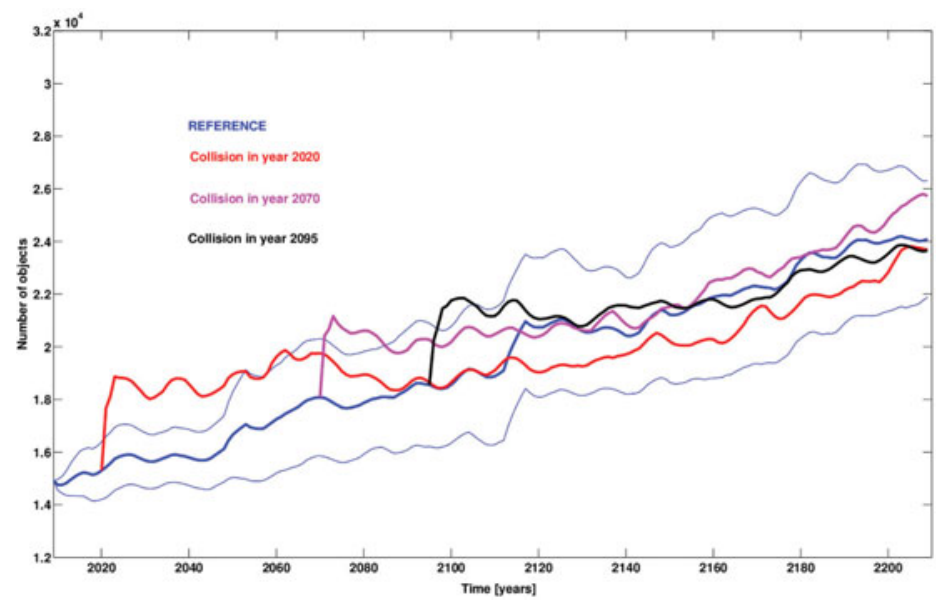

Figure 1. Number of objects larger than $10 \mathrm{~cm}$ in the reference scenario (blue line) and in 3 cases where a fragmentation of Envisat happens at three different epochs: 2020 (red line), 2070 (magenta line) and 2095 (black line). The thin blue lines represent the $\pm 1 \sigma$ standard deviation of the reference case.

parameters. The final goal is to measure the danger represented by typical classes of space objects, in order to be able to rank the abandoned space objects in terms of the possible effects on the environment of the spacecraft and, conversely, in terms of the effect of the environment on the spacecraft itself.

\section{Fragmentation ranking}

The full results of the above mentioned Contract are under final review and cannot be shown here, also for lack of space. Here we intend to present the analysis methods devised for this study, along with a few sample results.

Figure 1 shows the number of objects larger than $10 \mathrm{~cm}$ in LEO produced in the reference case (thick blue line), averaged over 50 Monte Carlo runs. The thin blue lines represent the $\pm 1 \sigma$ standard deviation of the Monte Carlo simulations. The other three curves represent the number of objects obtained in scenarios where the fragmentation of a spacecraft resembling the Envisat polar platform (mass $\simeq 8000 \mathrm{~kg}$ ) is artificially introduced in the simulation. In particular it was assumed that this additional fragmentation would take place in the year 2020 (red line) or in year 2070 (magenta line) or in year 2095 (black line). The Envisat fragmentation is supposed to take place in a decaying orbit, i.e., the same spacecraft is always fragmented but at different altitudes according to the epoch, from about $760 \mathrm{~km}$ of altitude in the year 2020 to about $695 \mathrm{~km}$ in 2095 .

Contrary to what one might expect, the final number of objects in all the four cases is statistically the same (i.e., all well within the $\pm 1 \sigma$ standard deviation bounds). This means that, in the long run, even the fragmentation of a very large spacecraft leaves no noticeable signature on the environment. The reason for this outcome is that the reference evolution is highly stochastic and is dominated by a large number of fragmentations (on average one every 5 years). Therefore, the effects of our additional Envisat-like fragmentation get soon "diluted" in the vast number of background fragments and leave almost no trace after 200 years. On the other hand, the situation can be different in the "interim" regime, in the orbital regions in the vicinity of the Envisat fragmentation, during the few decades following the event; these shorter term effects have been studied too and will be described elsewhere. 
In order to analyze many different long term evolution scenarios, it is necessary to find an evaluation norm to order the events in terms of their danger for the environment. For this purpose we devised a norm able to quantify and easily visualize these results. Given an underlying "reference" scenario and a "fragmentation" scenario in which the simulation of a particular fragmentation is added, the growth of the population of the "fragmentation" scenario w.r.t. the "reference" one can be quantified by:

$$
C_{i}=\left|\frac{n_{F R A G}(i)-n_{R E F}(i)}{\sigma_{R E F}}\right|
$$

where $n_{F R A G}$ is the number of objects in the fragmentation case, $n_{R E F}$ is the number of objects in the reference case and $\sigma_{R E F}$ is the standard deviation of the reference Monte Carlo runs.

As an example, Fig. 2 plots the value of $C_{i}$ in the case of the Envisat fragmentation scenarios shown in Fig. 1. The decreasing relative importance of the fragmentations happening in later years (both due to the larger number of "background" fragments and, mainly, to the lower altitude of the event) is clearly highlighted here.

A ranking of the danger represented by selected fragmentations can be easily expressed with a single number. In fact, the sum of the differences, weighted by the time interval, gives an indication of the criticality:

$$
C^{*}=\sum_{i=1}^{N} \frac{C_{i}}{N}=\sum_{i=1}^{N}\left|\frac{n_{F R A G}(i)-n_{R E F}(i)}{\sigma_{R E F}}\right| / N
$$

where $N$ is the number of years in the simulation. In the ESA study a full ranking of a large number of different fragmentations is being elaborated and will be soon published in a forthcoming paper.

\section{The Criticality of Spacecraft Index}

Similarly to what is currently done in the Near Earth Object field with the Palermo Scale (Chesley et al. 2002), a quantitative measure of the criticality of the artificial objects in Earth orbit would be important for a number of reasons. In particular, it would help

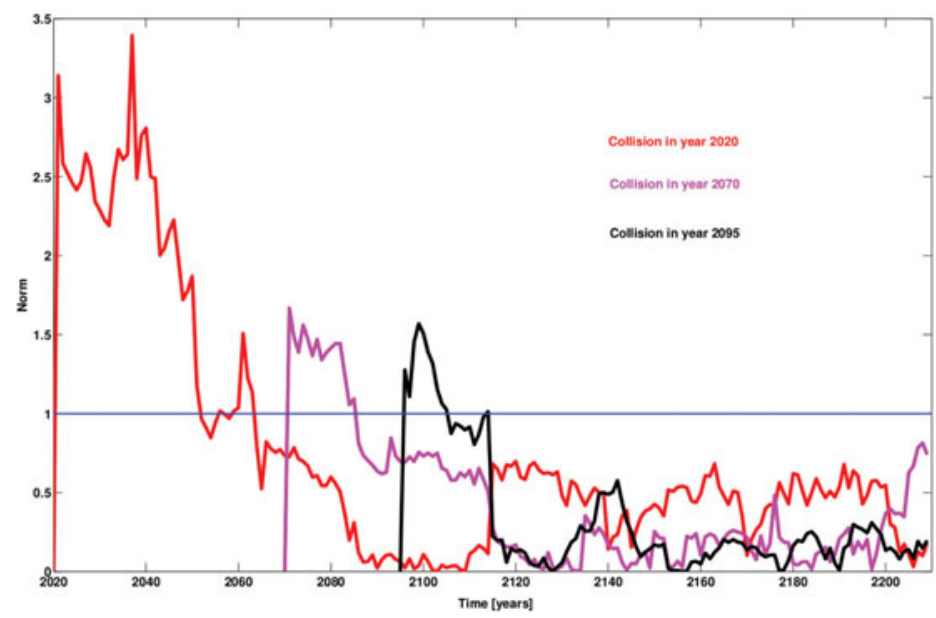

Figure 2. The time evolution of the norm of Eq. 2.1, computed for the three Envisat-like fragmentations, shown in Fig. 1. 


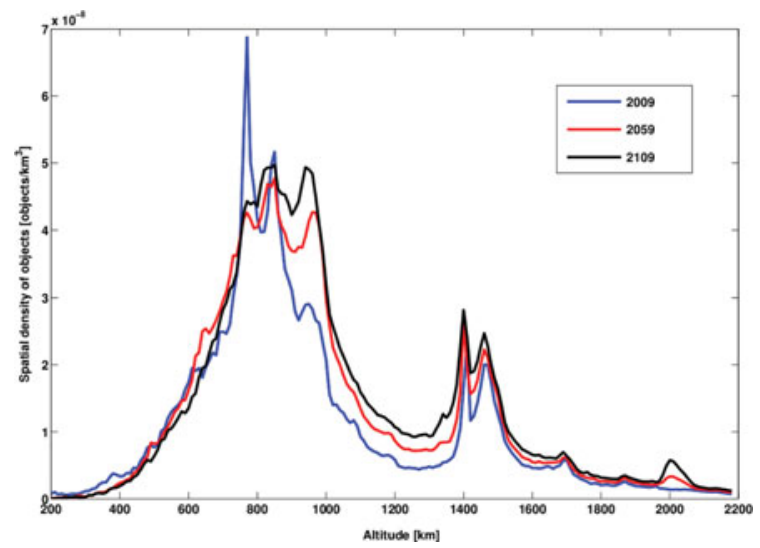

Figure 3. Spatial density of objects as a function of altitude in three different epochs: 2009 (blue line), 2059 (red line) and 2109 (black line).

spacecraft operators to easily assess the current collision risk faced by a given asset in space; moreover it would be a measure to evaluate the potential danger posed to the environment in case an object would be abandoned in space and therefore could be used as a ranking to establish active removal priorities. Finally, as is the case of the Palermo Scale, it could be useful to spread the public awareness of the danger posed by space debris by allowing a wider non-specialist audience to catch, with a single number, the environmental criticality of a given spacecraft.

For this purpose we developed an index, called the Criticality of Spacecraft Index (hereafter, CSI). In the following sections the factors entering in the definition of the CSI are described and discussed.

Environment dependence. The environment is considered in terms of the spatial density of objects as a function of time and altitude. It is well known that the collision probability is higher in regions where a higher concentration of objects is found. For this purpose, a reference simulation of the evolution of the space debris environment, spanning 200 years (considering the population of objects larger than $10 \mathrm{~cm}$ from the MASTER 2009 population), was performed with SDM 4.2 (Rossi et al. 2009).

For this reference case, a scenario where the space activities are performed in a way similar to the one adopted in the last decade is simulated. In particular, the traffic launch repeats an 8-year cycle representing the current launch pace, an 8-year lifetime is assumed for future spacecraft, no new explosions are considered and no avoidance maneuvers are performed. A post mission disposal scenario according to the 25-year rule is adopted, with a $60 \%$ compliance to this rule (i.e., only $60 \%$ of the spacecraft are actually deorbited at end-of-life). This reference scenario was simulated with 50 Monte Carlo runs and averages of the evolutions were computed. In particular, the resulting spatial density of objects as a function of altitude was recorded every year and stored. As an example, Fig. 3 shows the spatial density of objects larger than $10 \mathrm{~cm}$ as a function of altitude, for three different epochs.

The way in which the spatial density is taken into account in the CSI is as follows: given an epoch (or interval of time) and the orbital altitude, $h$, of the object under consideration, the spatial density, $D$, is taken from the stored values and normalized to the value of the maximal spatial density in the initial year 2009, that is the one at the altitude of $770 \mathrm{~km}, D_{0}$. Therefore, the multiplicative contribution to the CSI accounting 

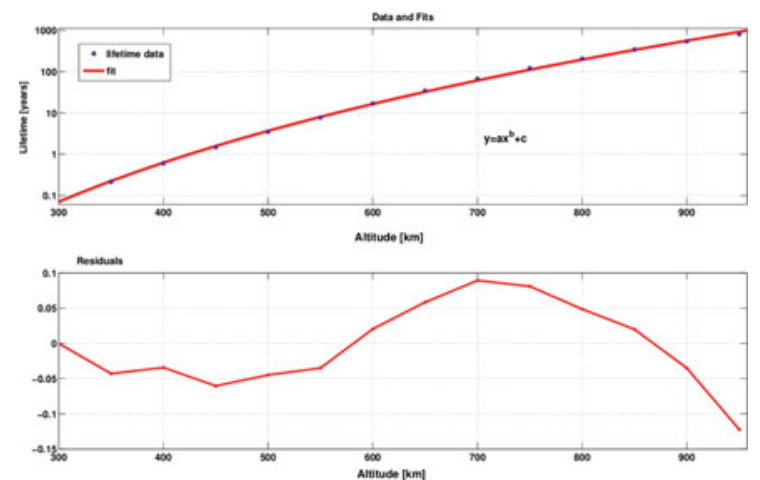

Figure 4. Orbital lifetime of a sample object with $A / M=0.012 \mathrm{~m}^{2} \mathrm{~kg}^{-1}$ as a function of altitude. The upper panel shows a power law fit to the lifetime values and the bottom panel shows the residuals of the fit (see text for details).

for the environment density is given by:

$$
\frac{D(h)}{D_{0}} .
$$

Lifetime dependence. The danger represented by an object left in space and the probability that it will be destroyed by a collision is a function of the time that this object will spend in space. Moreover the long term consequences of a fragmentation are much more severe for events happening at high altitudes where the cleaning effects of the atmosphere are not effective. Therefore the residual lifetime of an object is an important parameter to include in the index computation.

The lifetime of the objects, as a function of the orbital altitude $h$, is estimated from an average lifetime given by the curve shown in Fig. 4, computed assuming an area over mass ratio, $A / M=0.012 \mathrm{~m}^{2} \mathrm{~kg}^{-1}$, which reflects the average value observed for intact objects. An average solar flux between 110 and 130 units is considered. The lifetime curve was computed as a power law fit of the form:

$$
\log (\text { life })=a h^{b}+c
$$

where $a, b$ and $c$ are the coefficients of the fit. Therefore, given an object with mean altitude $h$ (note that, for LEO objects having low eccentricity, the semimajor axis can be used as a good approximation of $h$ ) the index term accounting for the lifetime is given by:

$$
\frac{\operatorname{life}(h)}{\operatorname{life}\left(h_{0}\right)}
$$

where $\operatorname{life}(h)$ is computed with Eq. 3.1 and the normalizing value is computed, as a default, for $h_{0}=1000 \mathrm{~km}$.

Mass dependence. Along with the altitude of the event, the other most influential parameter in determining the environment consequences of a given fragmentation is the mass, $M$. This fact is taken into account in the index by including the term:

$$
\frac{M}{M_{0}}
$$

where the normalizing factor is arbitrarily taken as $M_{0}=10000 \mathrm{~kg}$. An alternative explored is to use the same exponent found in the NASA breakup model by expressing 
the mass term as:

$$
\left(\frac{M}{M_{0}}\right)^{0.75}
$$

It has been checked that the adoption of the 0.75 exponent does not change significantly the results. Therefore, for the sake of simplicity, in the following the value of the exponent used is 1 .

Inclination dependence. It is well known that the collision risk is maximum for high inclination orbits that can cross all the other orbits in their range of altitude and that can lead to very high mutual inclinations (and therefore high impact velocities) due to the precessing orbital planes. For this reason an inclination $(i)$ dependence is included in the CSI, in the form:

$$
\frac{1+k \Gamma(i)}{1+k}
$$

where:

$$
\Gamma=\frac{(2-\cos (i))-1}{2}
$$

and $k=0.6$ since the typical flux of debris on an almost equatorial orbit is about $60 \%$ of the flux on a polar orbit.

Index definition. Combining the terms described above, the final definition of the CSI reads as:

$$
\Xi=\frac{M(h)}{M_{0}} \frac{D(h)}{D_{0}} \frac{\operatorname{life}(h)}{\text { life }\left(h_{0}\right)} \frac{1+k \Gamma(i)}{1+k},
$$

where we denote with $\Xi$ the value of the CSI. The definition was kept as simple as possible in order to allow its easy application and understanding by the largest possible community; the larger the value of the CSI, the more dangerous to the environment is an abandoned object.

Note that, due to the normalization, for all the space objects in our population $\Xi<1$, although in theory it is not bound by 1 . In order to consider possible time variations in the environment, the CSI could also be computed taking into account the average density of objects over an interval of time (e.g., 10 years) instead of the single value in the year of reference.

In order to get an idea of the values of the CSI that are to be expected, we list in Table 3 the 15 objects having the largest $\Xi$ in our population (MASTER 2009). Given the illustrative purpose of this discussion, in the Table are not listed "names" of objects, but rather physical and orbital characteristics. This allows to identify "families" of objects particularly dangerous for the environment and prone to potential active debris removal missions.

As it can be noticed, all the objects have large mass, well above one metric ton. However, it is also worth noting that the ranking is not just dominated by the mass, given that the semimajor axis (i.e., the mean altitude) plays a significant role, and that all the objects in the Table have high equatorial inclinations.

In Fig. 5 the objects having the highest 100 values of the CSI appear as circles with diameters proportional to their mass, and the panels show their distributions in terms of the mean altitude and inclination. In these planes the most critical objects can naturally be grouped into "families" according to their missions and launching countries.

It is worth noting how the orbital distributions shown in Figs. 5 compare nicely with Fig. 13 of Liou (2011), where the orbital distribution of the existing LEO R/Bs and S/Cs having highest mass and collision probability products (computed with 100 Monte Carlo runs of LEGEND) is shown. This confirms that the CSI can be considered as a reliable 
Table 1. List of the 15 objects having the largest values of the index in our MASTER 2009 population. Objects in boldface are upper stages, the others are satellites.

\begin{tabular}{|c|c|c|c|c|c|}
\hline \multicolumn{3}{|c|}{$\mathrm{a}[\mathrm{km}] \mid$ ecc } & inc & Mass $[\mathrm{kg}]$ & $\Xi$ \\
\hline 1 & 7372.2 & 0.002 & 99.25 & 9000.0 & 0.313 \\
\hline 2 & 7365.7 & 0.003 & 64. & 4500.0 & 0.163 \\
\hline 3 & 7343.1 & 0.003 & 64.99 & 4955.0 & 0.161 \\
\hline 4 & 7342.1 & 0.0 & 6 & 0 & 0.160 \\
\hline 5 & 7355.2 & 0.006 & 64.49 & 4500.0 & 0.154 \\
\hline 6 & 7346.5 & 0.007 & 65. & 4500.0 & 0.151 \\
\hline 7 & 7342.9 & 0.006 & 64.95 & 4500.0 & 0.146 \\
\hline 8 & 7349.3 & 0.005 & 64 & 4500.0 & 0.145 \\
\hline 9 & .1 & 0 . & & & 0.143 \\
\hline 10 & 7222.0 & 0.0 & 71 & 9000.0 & 0.139 \\
\hline 11 & 7221.6 & 0.000 & 70.98 & 9000.0 & 0.139 \\
\hline 12 & 7336.6 & 0.004 & 64.70 & 4500.0 & 0.135 \\
\hline 13 & 7227.3 & 0.002 & 70.88 & 8226.0 & 0.135 \\
\hline 14 & 7335.5 & 0.006 & & 4500.0 & 0.134 \\
\hline 15 & 7333.3 & 0.009 & 65.08 & 4500.0 & 0.131 \\
\hline
\end{tabular}
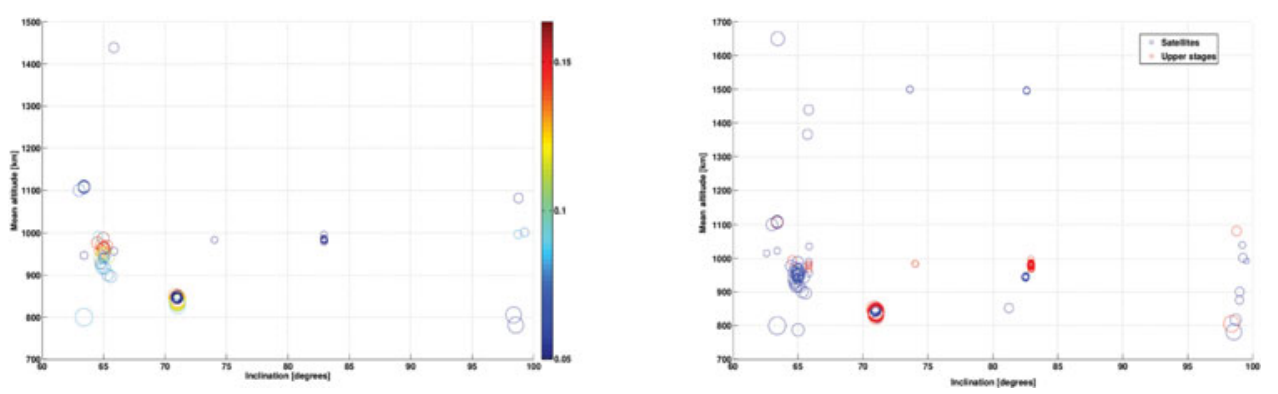

Figure 5. Distribution of the first 100 objects in the index ranking as a function of inclination and of the mean altitude. In the left panel, the color of the circles gives $\Xi$, according to the color coding on the right. In the right panel, the blue circles represent satellites while the red ones represent upper stages. The size of the circles is proportional to the mass of the object.

indicator of the actual risk faced and posed by objects in LEO and as such is a good analytic, fast and easy-to-compute proxy for active removal strategies planning.

The histograms in Fig. 6 show the distribution in altitude and inclination, respectively, of all the fragmentations recorded in the 50 Monte Carlo runs performed with SDM to derive the spatial density distributions. Therefore these distributions highlight the most dangerous zones in LEO in terms of the catastrophic collision risk. Within these histograms the red dots show the orbital parameters of the top 500 objects in the CSI ranking. It can be noticed how these objects clearly populate the bins where the highest number of collisions occur, once again showing that the CSI is a good proxy for the collision risk faced by a spacecraft in LEO.

We can conclude that the Criticality of Spacecraft Index presented in this paper is a simple, fast, easy-to-compute analytic tool able to rank the abandoned space objects in terms of the danger they can pose to the environment (or, conversely, in terms of the risk they face from the environment), taking into account their orbital and physical characteristics. Moreover, it is able to catch the main known features of the in-orbit collision risk and can be viewed as a good proxy to prioritize active debris removal planning.

\section{Acknowledgements}

The study described in the paper was performed in the framework of the contracts: SPARC-Space Threats and Critical Infrastructures: Risks and Countermeasures 

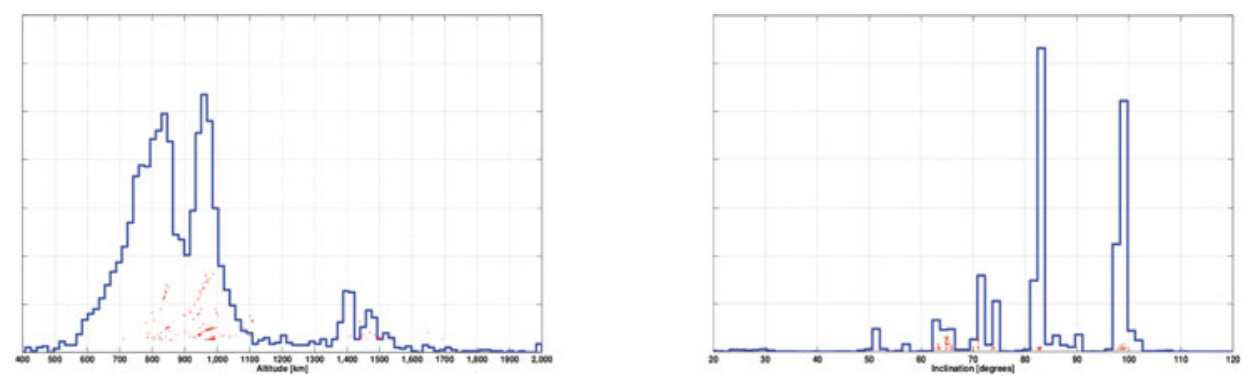

Figure 6. Altitude (left panel) and inclination (right panel) relative distribution of all the fragmentations recorded in the SDM runs of the reference case used to derive the objects spatial density. The red dots show the altitude and inclination distribution of the first 500 objects in the CSI ranking.

(HOME/2011/CIPS/AG/4000002119) and Assessment Study for Fragmentation Consequence Analysis for LEO and GEO Orbits, ESA/ESOC No. 4000106534/12/F/MOS. The authors wish to thank L. Anselmo and C. Pardini for useful discussions regarding the index computation.

\section{References}

Chesley, S. R., Chodas, P. W., Milani, A., Valsecchi, G. B. \& Yeomans, D. K. 2002, Icarus, 159, 423

Liou, J.-C. 2011, Advances in Space Research, 47, 1865

Rossi, A., Anselmo, L., Pardini, C., Jehn, R., \& Valsecchi, G. B. 2009, Proceedings of the Fifth European Conference on Space Debris, ESA SP-672, (ESA Communication Production Office, Noordwijk, The Netherlands) 